\title{
Transition from 1D to 2D laser-induced ultrasonic wave propagation in an extended plate
}

\author{
Jernej Laloš · Tomaž Požar · Janez Možina
}

Received: date / Accepted: date

\begin{abstract}
Optodynamic interaction between a laser pulse and the surface of an opaque, solid elastic object produces transient waves that propagate and reverberate within the object. They can be, in general, categorized into three distinctive types which are all formed through different mechanisms: ablation-induced waves (AIWs), lightpressure-induced waves (LIWs), and thermoelastic waves (TEWs). In this paper, out-of-plane displacements of such waves are simulated at the epicentral position on the opposite side of an extended plane-parallel elastic plate. Wave propagation is mathematically described by Green's transfer functions convolved with suitable time profiles of the incoming laser pulses. The simulated size of the circularly-symmetric laser illuminated area on the plate surface is varied to show the limit-to-limit transition of the displacement waveforms: from a 2D point source to an infinite 1D source.
\end{abstract}

Keywords Ablation · Elastic waves - Laser induced ultrasound · Optodynamics · Radiation pressure $\cdot$ Thermoelasticity

\section{Introduction}

Laser pulses are nowadays commonly used to induce elastic material waves in opaque solid objects [1-3]. Depending on the intensity level of the incident light pulse and on the material properties of the illuminated surface, different ultrasound-generation mechanisms give rise to elastic waves with different properties [4]. Once a certain fluence threshold of evaporation is exceeded, the ultrasound with the largest amplitude is usually generated by (confined) ablation when the expelled material is compensated by generation of ablation-induced waves (AIWs). The ubiquitous thermoelastic waves (TEWs) are generated due to absorption and subsequent thermal expansion. The elastic waves with the smallest amplitudes originate solely from the photon momentum transfer whenever the incident light is reflected, scattered or absorbed.

J. Laloš $(\varangle) \cdot$ T. Požar · J. Možina

University of Ljubljana, Faculty of Mechanical Engineering, Aškerčeva 6, 1000 Ljubljana, Slovenia E-mail: jernej.lalos@fs.uni-lj.si 
The latter, dubbed the light-pressure-induced waves (LIWs), have recently been experimentally observed [5]. Their properties are closely related to AIWs [4], except for a significantly smaller amplitudes. From the perspective of research areas of laser propulsion [6] and optical manipulation [4,7,8], AIWs and LIWs are monopolar waves and are capable of displacing the center-of-mass of the illuminated solid object, while TEWs, with a di-polar nature, carry a zero total momentum and can only perturb particles within the object around their initial equilibrium positions.

Experimentally, these types of waves can be studied separately. LIWs are best observed in low-loss mirrors with high-reflectivity dielectric coatings along with a laser fluence well below laser-induced damage threshold. For TEWs, a light-absorbing material needs to be used requiring a laser fluence below ablation threshold. While AIWs are best generated with a high-intensity laser pulse whose fluence exceeds the ablation threshold by a large margin [9]. Practically, a combination of wave types is induced at the same time by a single laser pulse when conditions do not meet the extremes presented above $[4,10,11]$.

When a physical system is idealized to the extent to render it amenable to theoretical treatment, the dimensionality is often reduced so that fewer dimensional variables are present in the (thermo)elastic governing equations and their solutions $[1,2,12]$. The simplest and very illustrative is the one-dimensional (1D) treatment, where a light pulse with plane wavefronts and infinite lateral extent impinges perpendicularly onto an extended plane-parallel elastic plate. In this case, the only displacement component of the material due to the presence of light-induced elastic waves is in the direction parallel with the direction of laser-pulse propagation. Closed-form analytical solutions of this problem for plate geometry were found theoretically for ablative or radiation pressure [4,13] and thermoelastic generation [14-17]. This regime can be reached experimentally with homogeneous pulsed illumination, such as with laser pulses featuring top-hat profiles, and is valid until elastic waves that originate from the edge of the interaction area reach the sensor. On the other hand, if the laser pulse is tightly focused on the top surface of the plate, the problem can be satisfactorily modeled with a point interaction. This point-source problem is two-dimensional (2D). It has an additional degree of freedom which is the displacement in the radial direction. In this case, the solution of the governing elastodynamic equation, called the Green's function [18-20], can be used to form arbitrary spatial source distributions similar to Huygens-Fresnel wave propagation principle [21]. All generation regimes can be modeled with Green's functions. For ablative and radiation pressure regime, the normal force input Green's function is used [10,11,22], while thermoelastic source is modeled with isotropic force dipoles [3, 10, 11,23].

The waveforms due to AIWs, TEWs or LIWs are so dissimilar when homogeneous (1D) source and point source (2D) are compared that the gradual transition from one limiting case to the other would facilitate further understanding of the laser-induced wave propagation in plates and other bounded solids. Thus, the main goal of this paper is to present this transition based on the simulated waveforms obtained when the wave-generating force is normal to the illuminated surface, such as with AIWs and LIWs, and when it can be thought of as material expansion giving rise to TEWs. The transition is modeled with an expanding circular area of homogeneous illumination, starting with a point, representing a focused laser pulse, and finishing with the radius 
much larger than the thickness of the plate. Since most sensors are sensitive only to out-of-plane displacements $[24,25]$ and they can only measure the displacements of the surface of an object, only the normal surface displacements is calculated. The results additionally illuminate the comparison between the epicentral waveforms in both limiting cases of the thermoelastic regime - between the compression of the first $\mathrm{P}$-wave arrival in the 1D geometry and the dominant rarefaction of the same transient in the point source $2 \mathrm{D}$ limit.

\section{Waveform simulation}

The simulation is based on the Green's function formalism coupled with a statistically derived weight function, which is extensively expounded in [21]. It simulates the out-of-plane displacement waveforms at the epicentral point on the plate surface that were induced by a circularly-symmetric laser pulse incident on the opposite surface of the plate for different sizes of its impact area and different generation regimes.

A set of wave equations and boundary conditions describes wave propagation in matter $[1,12]$. Their solutions, for a $\delta$-function input impulse, are Green's functions $g\left(\boldsymbol{r}_{0}, \boldsymbol{p}_{0}, t\right)[19,20]$. It is interpreted that they transform an input signal, such as force impulse $f\left(\boldsymbol{r}_{0}, t\right)=J \delta\left(\boldsymbol{r}_{0}\right) \eta(t)$, at one point $\boldsymbol{r}_{0}$, to an output signal, such as displacement waveform $u\left(\boldsymbol{p}_{0}, t\right)$, at another point $\boldsymbol{p}_{0}$. Schematics of this geometric arrangement is presented in Fig. 1(a). Such transformation can be expressed as a temporal convolution between the appropriate Green's function and the time profile $\eta(t)$ of the force impulse:

$$
u\left(\boldsymbol{p}_{0}, t\right)=J \int_{-\infty}^{\infty} g\left(\boldsymbol{r}_{0}, \boldsymbol{p}_{0}, \tau\right) \eta(t-\tau) \mathrm{d} \tau,
$$

with $J$ representing the magnitude of the force impulse. Green's functions are specific for different elastic and geometric properties of each material, for different input and output signal locations and directions.

To simplify the modeling and make experiments comparable, a single plate substrate is used while its surface coatings are changed to accommodate different generation regimes. For example, a low-loss high-reflectivity surface coating can be used to enhance LIWs and suppress TEWs, while a metal surface coating can be employed to foster TEWs just under the plate surface.

Since AIWs and LIWs have the same propagation mechanisms, though different generation mechanisms, only the LIWs are going to be modeled further on. The LIWs are induced by light pressure which acts normally on the plate surface. Since only out-of-plane displacements are of concern, only the $g_{33}$ type of Green's functions, in which both input force impulse and output displacement are normal to the plate surfaces, needs to be used [21].

The TEWs are induced by thermal expansion of matter due to light absorption. Since thermal expansion is isotropic, three types of Green's functions $g_{311}, g_{322}$, and $g_{333}$ need to be superimposed over each-other to represent the point-expansion $[3,23]$. These are force di-poles and, for each of them, the input force couple acts in one of 


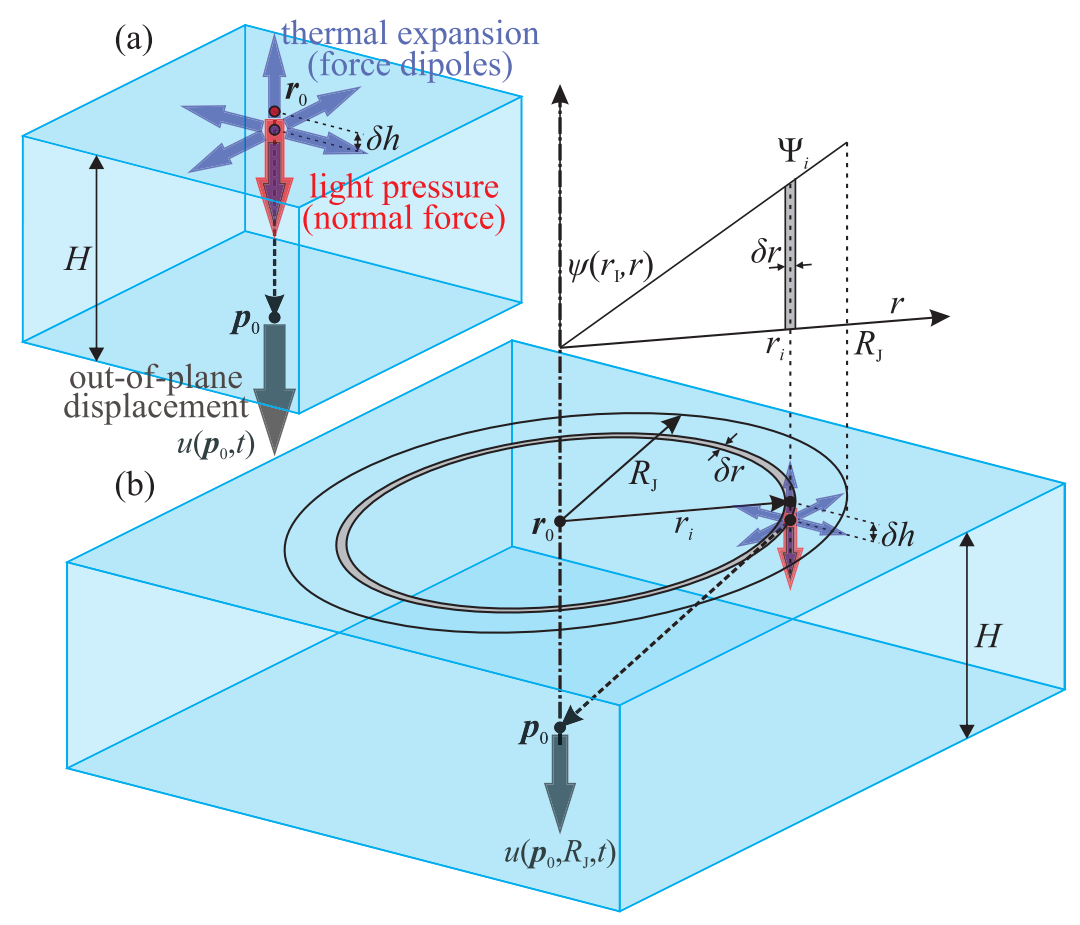

Fig. 1 Schematics of general geometric arrangement for the (a) point-to-point and (b) area-to-point models

the three principal directions while, for all of them, the output displacement occurs in the direction normal to the plate surface.

Though light absorption decreases exponentially with depth $h$ according to BeerLambert law, an approximation is made to simulate the effect of light absorption just beneath the plate surface. In it, the source point is taken as buried below the impact surface at the average depth of optical penetration $\delta h=h_{0} \ln (2)$, where $h_{0}$ is the penetration depth, meaning that half of the pulse energy is absorbed above $\delta h$ and the other half below it. Since thermal expansion is rapid, while contraction due to cooling occurs on larger time scales, contractions can be neglected. Thus, to simulate thermal expansion, these Green's functions have to be additionally convolved with a step function.

Such a model works well only in the point-source limit. It has to be expanded, however, in order to simulate the expansion of the laser pulse impact area [21,25]. To cover a larger impact area with a diameter of $R_{\mathrm{J}}$ and centered at a point $\boldsymbol{r}_{0}$, a special area-to-point (AP) transfer function $g_{\mathrm{AP}}\left(\boldsymbol{r}_{0}, \boldsymbol{p}_{0}, R_{\mathrm{J}}, t\right)$ has to be constructed. In the interest of numerical calculations performed here, the in-plane radial distance component $r$ running from the center of the impact area $\boldsymbol{r}_{0}$ towards its edge at $R_{\mathrm{J}}$ on the impact surface has to be discretized into radial distance points $r_{i}$, as indicated in Fig. 1(b). These discrete points are taken as new input positions for the calculation of further Green's functions $g\left(\boldsymbol{r}_{0}, \boldsymbol{p}_{0}, r_{i}, t\right)$ needed in this expanded model while their 
output point remains the same epicentral point $\boldsymbol{p}_{0}$. The AP transfer function is thus calculated by superimposing the appropriately weighted Green's functions:

$$
g_{\mathrm{AP}}\left(\boldsymbol{r}_{0}, \boldsymbol{p}_{0}, R_{\mathrm{J}}, t\right)=\sum_{i} \Psi_{i} g\left(\boldsymbol{r}_{0}, \boldsymbol{p}_{0}, r_{i}, t\right)
$$

Since the neighboring radial distance points are equidistant from each-other by $\delta r=$ $r_{i+1}-r_{i}$, the discretization has to be fine enough to form a smooth superposition of weighted Green's functions.

The individual weights $\Psi_{i}$ are calculated as an integral of the normalized weight function $\psi\left(R_{\mathrm{J}}, r\right)$ with the integration interval centered at the radial distance point $r_{i}$ and the width of $\delta r$ :

$$
\Psi_{i}=\int_{r_{i}-\delta r / 2}^{r_{i}+\delta r / 2} \psi\left(R_{\mathrm{J}}, r\right) \mathrm{d} r
$$

In this case, the weight function depends on the size $R_{\mathrm{J}}$ of the impact area and the intensity profile of the laser pulse. For a uniform laser pulse profile, the weight function is proportional to the radius: $\psi\left(R_{\mathrm{J}}, r\right)=2 r / R_{\mathrm{J}}^{2}$, where it is defined: $0 \leq r \leq R_{\mathrm{J}}$.

The appropriate directional Green's functions $\left(g_{33}\right)$ in the case of LIWs and AIWs and the superposition of their spatial derivatives $\left(g_{311}+g_{322}+g_{333}\right)$ in the case of TEWs have to be used in Eq. (2), as described for the point-source limit [3].

Similarly as in the PP model, a temporal convolution of the AP transfer function and the time profile $\eta(t)$ of the incident laser pulse has to be performed to obtain the out-of-plane displacement waveform:

$$
u\left(\boldsymbol{p}_{0}, R_{\mathrm{J}}, t\right)=J \int_{-\infty}^{\infty} g_{\mathrm{AP}}\left(\boldsymbol{r}_{0}, \boldsymbol{p}_{0}, R_{\mathrm{J}}, \tau\right) \eta(t-\tau) \mathrm{d} \tau,
$$

with $J$ representing the magnitude of the laser force impulse.

Green's functions used in the simulation are numerically calculated using a modified algorithm developed by Hsu [19] for a plane-parallel extended plate.

The waveforms are simulated on a commonly used plane-parallel glass plate made of UV-grade fused silica $\left(\mathrm{SiO}_{2}\right)$. Its thickness is set at $H=10 \mathrm{~mm}$, its mass density at $\rho=2200 \mathrm{~kg} / \mathrm{m}^{3}$, Young's modulus at $Y=72 \mathrm{GPa}$ and Poisson's ratio at 0.17 . From these, the P-wave (compression) velocity $c_{\mathrm{P}}=5931 \mathrm{~m} / \mathrm{s}$ and the $\mathrm{S}$-wave (shear) velocity $c_{\mathrm{S}}=3740 \mathrm{~m} / \mathrm{s}$ of the plate are calculated [1]. For TEW simulation, the average depth of optical penetration is set at $\delta h=10 \mu \mathrm{m}$. This is viable as the waveform broadening effects of a couple of nanoseconds due to the exponential energy absorption are completely dominated by the pulse duration convolution, especially if the pulse lasts at least a few tens of nanoseconds [18].

The simulated laser pulse is circularly symmetric with a uniform intensity profile and a bell-shaped time profile with a full-width-at-half-maximum of $\Delta t=40 \mathrm{~ns}$. It impacts on a circular area which has a center at a point $\boldsymbol{r}_{0}$ on the plate surface and a radius of $R_{\mathrm{J}}$. To show the transition of the out-of-plane displacement waveforms from the point-source limit toward the infinite-source limit, the radii are varied: $R_{\mathrm{P}} \rightarrow 0$, $R_{\mathrm{I}}=1 \mathrm{~mm}, R_{\mathrm{II}}=2 \mathrm{~mm}, R_{\mathrm{III}}=3 \mathrm{~mm}, R_{\mathrm{IV}}=4 \mathrm{~mm}, R_{\mathrm{V}}=7 \mathrm{~mm}, R_{\mathrm{VI}}=10 \mathrm{~mm}$, 
$R_{\mathrm{VII}}=15 \mathrm{~mm}, R_{\mathrm{VIII}}=20 \mathrm{~mm}, R_{\mathrm{IX}}=35 \mathrm{~mm}, R_{\mathrm{X}}=50 \mathrm{~mm}$. The largest radius is large enough to be considered infinitely large during the extent of the simulation: $R_{\mathrm{X}} \rightarrow \infty$. Obviously, in a point-source limit with $R_{\mathrm{P}} \rightarrow 0$, its spatial distribution takes the form of a $\delta$-function. The simulated laser pulse has two different energy regimes: in one, the laser pulse energy remains constant $(E=$ const.) for all radii, while in the other, the fluence (energy density) is kept constant $\left(E / R_{\mathrm{J}}^{2}=\right.$ const.) for all radii.

The simulation lasts $t_{\Omega}=8.0 \mu \mathrm{s}$ from the start of the laser pulse impact on the plate surface while the sampling period is $\delta t=2 \mathrm{~ns}$. The duration of the simulation is sufficiently long to show those waves that are arriving directly from the source area to the epicentral point and the waves that have been reflected twice - from both surfaces - on their way from the source area to the epicentral point.

\section{Results}

The results of the simulation are presented in Figs. 2 and 3. The epicentral position displacement waveforms of TEWs for constant laser pulse fluence are shown in Fig. 2(a) and for constant laser pulse energy are given in Fig. 2(b). Similarly, the epicentral position displacement waveforms of LIWs (and AIWs) for constant laser pulse fluence are depicted in Fig. 3(a) and for constant laser pulse energy are shown in Fig. 3(b). Although all displacement waveforms are proportional within each plot, their displacement scales are arbitrary.

\section{Discussion}

Every source point in a source area produces, in general, various bulk and surface waves with each having its distinct propagation velocity [3]. Since the observation point is on the opposite side of the source, Rayleigh surface waves are not present in the simulated waveforms, while the arrivals of P-waves (longitudinal), S-waves (shear), and head waves give rise to distinct features in the out-of-plane displacement waveforms. During the propagation of the bulk waves within a plate, however, they may be mode-converted when they are reflected from its surfaces.

The LIW, AIW and TEW waveforms are presented in both energy regimes, with constant laser pulse fluence in Figs. 2(a) and 3(a) and with constant laser pulse energy in Figs. 2(b) and 3(b).

Each TEW waveform plot in Fig. 2 remains unperturbed until a relatively small positive peak, known as the precursor, starts to occur at the time $t_{\mathrm{P} 0}=H / c_{\mathrm{P}}=$ $1.69 \mu \mathrm{s}$. This is a P-wave generated by thermal expansion in the normal direction $\left(g_{333}\right.$ component) arriving along the shortest path directly from under the central point $\boldsymbol{r}_{0}$. It is followed immediately by a sharp negative step which is produced by $\mathrm{P}$-waves due to lateral thermal expansion $\left(g_{311}+g_{322}\right.$ components). A gentler negative ramp is then reversed sharply by a positive step with the arrival of the $S$-wave from the edges of the impact area. The shortest S-wave arrival time $t_{\mathrm{S} 0}=H / c_{\mathrm{S}}=2.67 \mu$ s occurs only in the PP-limit model, because S-wave arrives directly from under the central 


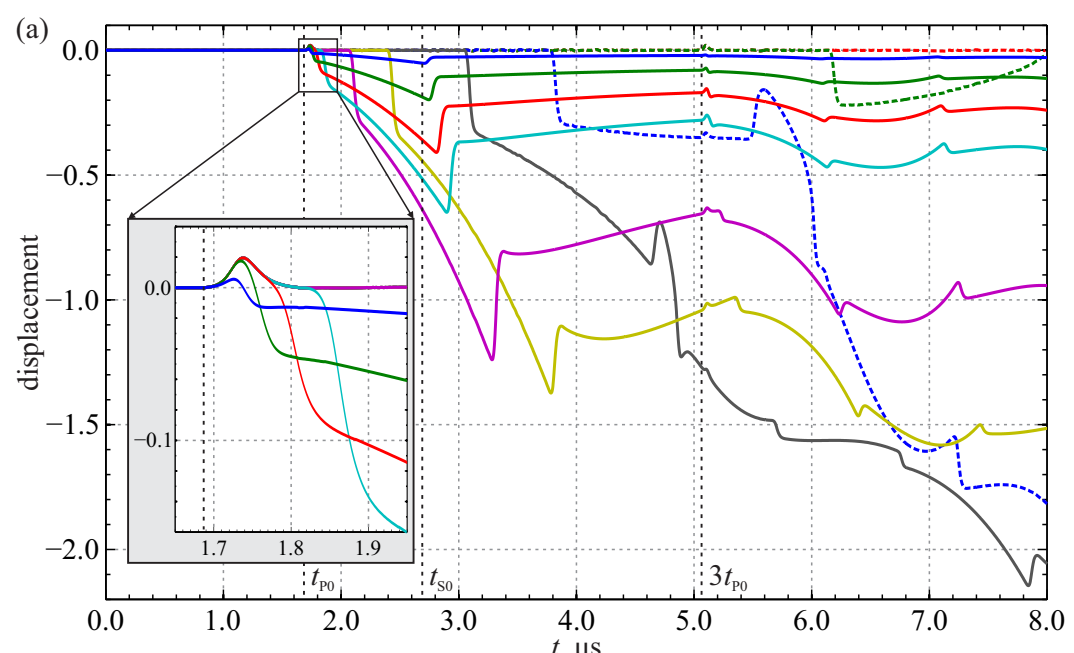

$$
\begin{array}{lll}
-u\left(\boldsymbol{p}_{0}, R_{\mathrm{I}}, t\right) ; R_{\mathrm{I}}=1 \mathrm{~mm} & -u\left(\boldsymbol{p}_{0}, R_{\mathrm{V}}, t\right) ; R_{\mathrm{V}}=7 \mathrm{~mm} & --u\left(\boldsymbol{p}_{0}, R_{\mathrm{IX}}, t\right) ; R_{\mathrm{IX}}=35 \mathrm{~mm} \\
-u\left(\boldsymbol{p}_{0}, R_{\mathrm{II}}, t\right) ; R_{\mathrm{II}}=2 \mathrm{~mm} & -u\left(\boldsymbol{p}_{0}, R_{\mathrm{VI}}, t\right) ; R_{\mathrm{VI}}=\mathrm{h}=10 \mathrm{~mm} & =-u\left(\boldsymbol{p}_{0}, R_{\mathrm{X}}, t\right) ; R_{\mathrm{X}}=50 \mathrm{~mm} \\
-u\left(\boldsymbol{p}_{0}, R_{\mathrm{III}}, t\right) ; R_{\mathrm{III}}=3 \mathrm{~mm} & -u\left(\boldsymbol{p}_{0}, R_{\mathrm{VII}}, t\right) ; R_{\mathrm{VII}}=15 \mathrm{~mm} & =-u\left(\boldsymbol{p}_{0}, t\right) ; R_{\mathrm{P}} \rightarrow 0 \\
-u\left(\boldsymbol{p}_{0}, R_{\mathrm{IV}}, t\right) ; R_{\mathrm{IV}}=4 \mathrm{~mm} & --u\left(\boldsymbol{p}_{0}, R_{\mathrm{VIII}}, t\right) ; R_{\mathrm{VIII}}=20 \mathrm{~mm} &
\end{array}
$$

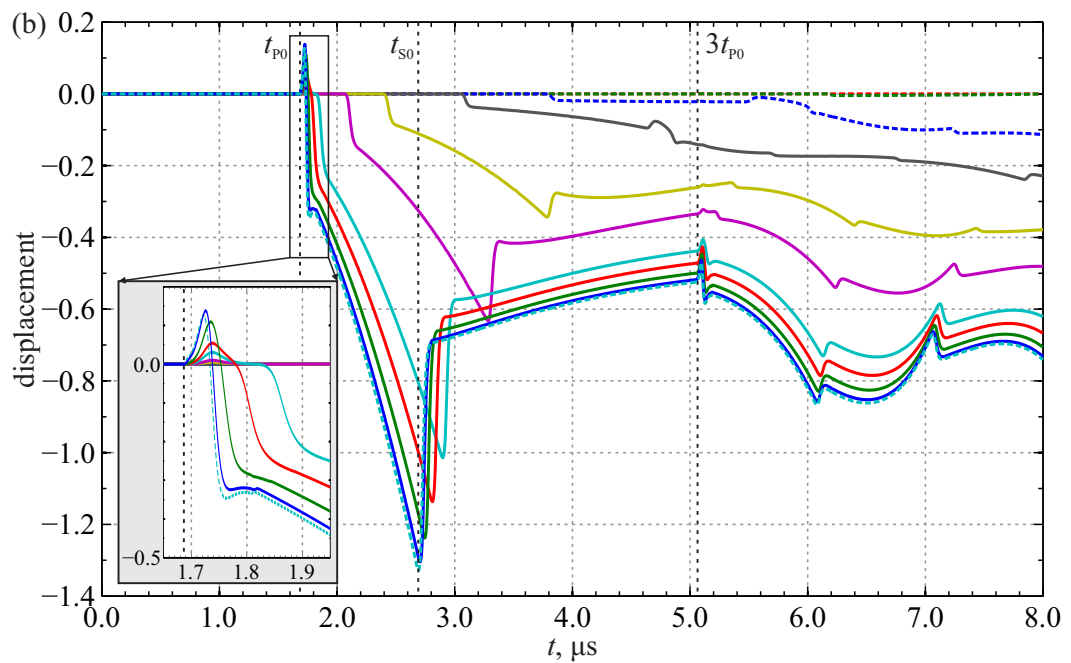

Fig. 2 The epicentral position displacement waveforms of thermo-elastic waves for different impact area radii (a) at constant laser pulse fluence and (b) at constant laser pulse energy

point $\boldsymbol{r}_{0}$. Another relatively small peak starts rising at the time of $3 t_{\mathrm{P} 0}=5.06 \mu \mathrm{s}$. It is a twice reflected PPP-wave arrival which has traveled three times along the shortest path between the $\boldsymbol{r}_{0}$ and the $\boldsymbol{p}_{0}$. Other distinct features in the waveforms pertain to multiply-reflected and mode-converted waves. A similar modeling of the thermoelastic out-of-plane displacement waveforms up to the first S-wave arrival was also performed by Every et al. [26]. 

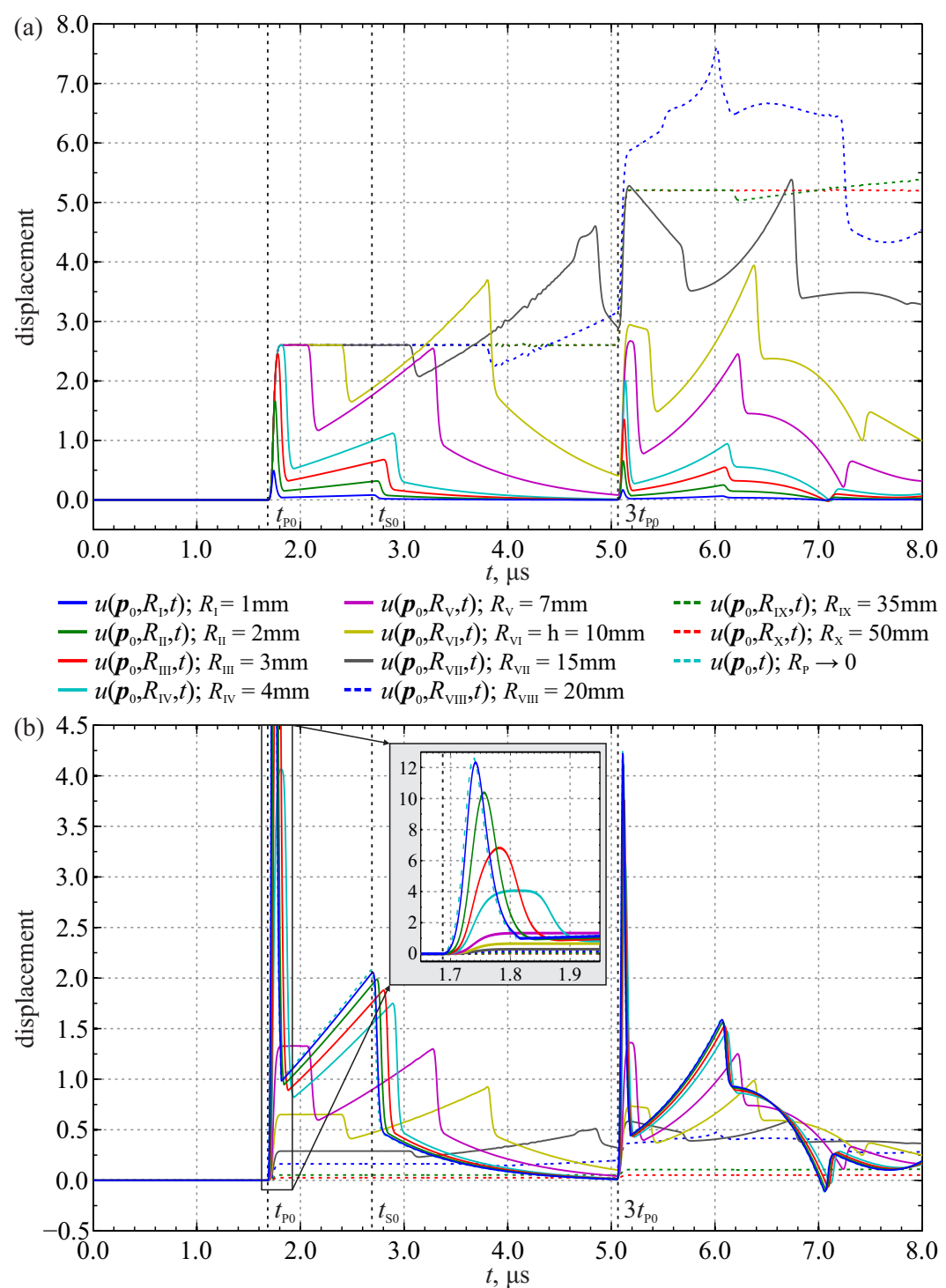

Fig. 3 The epicentral position displacement waveforms of light-pressure-induced waves (and, quite similarly, for ablation-induced waves) for different impact area radii (a) at constant laser pulse fluence and (b) at constant laser pulse energy

While following the limit-to-limit transition, an unperturbed part of the waveform starts to occur after the initial positive peak when the impact area reaches a certain size. This flat part is due to the fact that $\mathrm{S}$-waves originating from inside of the impact area cancel each-other out so that only the P-waves (negative steps) and S-waves (positive steps) originating from the edge of the impact area reach the epicentral 
point. As the impact area grows larger, the unperturbed waveform grows with it, until, in the 1D limit, almost entire waveform is flat with only P-wave positive peaks rising from it. These peaks are consistently occurring at times of $t_{\mathrm{P} j}=(2 j-1) t_{\mathrm{P} 0}$, where $j=1,2,3 \ldots[17,27]$.

The peaks in otherwise unperturbed waveform in the 1D limit demonstrate the fact that TEWs do not add momentum to the object and cannot move its center of mass.

In Fig. 3, each LIW (or AIW) waveform plot remains unperturbed until a relatively large positive peak starts to occur at the time $t_{\mathrm{P} 0}$. This is, similarly as with TEWs, a P-wave arriving along the shortest path directly from the central point $\boldsymbol{r}_{0}$. A gentler rising ramp is reversed sharply with the arrival of the $\mathrm{S}$-wave from the edges of the impact area. The shortest $S$-wave arrival time $t_{\mathrm{S} 0}$ occurs, as with TEWs, when the $S$-wave arrives directly from the central point $\boldsymbol{r}_{0}$ in the PP-limit model. Another relatively large peak starts rising at a time of $3 t_{\mathrm{P} 0}$. This is a twice reflected PPP-wave arrival, similarly as with TEWs.

While following the limit-to-limit transition, a flat step starts to form after the first $\mathrm{P}$-wave positive peak when the impact area reaches a certain size. The length of the flat plateau is the same as the length of the zero-displacement interval in the TEW waveforms. This flat part occurs due to the fact that $\mathrm{S}$-waves originating from inside of the impact area cancel each-other out, while the direct P-waves are still arriving from other, more distant, parts of the impact area. As the impact area grows larger, the flat steps in the waveform grow with it, until, in the 1D limit, the entire waveform consists of discrete flat steps that are periodically occurring at times of $t_{\mathrm{Pj}}[28,29]$.

The flat steps in the 1D limit demonstrate the fact that LIWs and AIWs add momentum to the object and move its center of mass, thus producing macroscopic motion.

Comparing both figures, it can be seen that, given a constant laser pulse energy, the largest displacement amplitude is achieved with the most focused pulse.

\section{Conclusions}

Laser-pulse induced ultrasound was modeled for three different generation regimes: ablation, thermoelastic and radiation pressure. The normal displacement at the epicentral position on the opposite side of the illuminated area on the plane-parallel extended plate was calculated for circularly-symmetric, top-hat interaction profiles by varying the radius of the source from the $2 \mathrm{D}$ point-source limit to the infinite and uniform 1D limit. The theoretical approach was based on the Green's function formalism, where point radiators of ultrasound where collected to form a wide, top-hat source. Temporal distribution of the source was achieved through numerical convolution.

The results of the modeling coincide with the already known waveforms for both limiting cases, while the intermediate displacement histories supplement the knowledge of the not-so-intuitive transition between them. The waveforms caused by the arrivals of the ablation-induced waves (AIWs) and light-pressure induced waves (LIWs) have identical shapes, although their amplitudes may vary by several orders of magnitude for the same laser pulse but different surface properties of the plate. Here, 
the development of the epicentral displacements with increasing source radius shows how shear waves cancel out, leaving, in the 1D limit, only a single mono-polar wave which reverberates within the plate and causes a distinctive staircase-like rear surface displacement. Likewise, shear waves also nullify in the thermoelastic regime, leaving in the 1D limit a single, di-polar echoing wave. In contrast, TEWs cannot propel the plate and so the rear surface only experiences discrete and transient outward bulges occurring periodically at each arrival of the TEW to the epicentral position.

The results find their applications not only in laser ultrasonics, but also in the modeling and understanding of elastic waves generated by circular piezoelectric actuators. A similar simulation approach can also be used in the studies of acoustic emission and tremors due to impacts of celestial or man-made objects with Earth's crust.

Acknowledgements The research was conducted as a part of the Optodynamics programme (P2-0392), in duration from 2015-1-1 to 2019-12-31, financed by the Slovenian Research Agency.

\section{References}

1. C.B. Scruby, L.E. Drain, Laser Ultrasonics: Techniques and Applications (Adam Hilger, Bristol [etc.], 1990)

2. S.J. Davies, C. Edwards, G.S. Taylor, S.B. Palmer, J. Phys. D Appl. Phys. 26, 329 (1993)

3. D. Royer, E. Dieulesaint, Elastic Waves in Solids II: Generation, Acousto-optic Interaction, Applications (Springer-Verlag, Berlin, 2000)

4. T. Požar, A. Babnik, J. Možina, Opt. Express 23, 7978 (2015)

5. T. Požar, J. Možina, Phys. Rev. Lett. 111, 185501 (2013)

6. J. Možina, T. Požar, In High Power Laser Ablation and Beamed Energy Propulsion: HPLA/BEP, ed. by C. Phipps (Cvent, Santa Fe, 2014), p. 1

7. T. Požar, J. Možina, Proc. SPIE 9548 (2015), 954800

8. D.G. Grier, Nature 424, 810 (2003)

9. R.J. Dewhurst, D.A. Hutchins, S.B. Palmer, C.B. Scruby, J. Appl. Phys. 53, 4064 (1982)

10. B. Mi, I.C. Ume, J. Nondestruct. Eval., 21, 23 (2002)

11. J.-D. Aussel, J.-P. Monchalin, Ultrasonics 27, 165 (1989)

12. J.D. Achenbach, Wave Propagation in Elastic Solids (North-Holland Publishing, Amsterdam, 1999)

13. T. Požar, J. Možina, Opt. Lett. 38, 1754 (2013)

14. R.M. White, J. Appl. Phys. 34, 3559 (1963)

15. J.C. Bushnell, D.J. McCloskey, J. Appl. Phys. 39, 5541 (1968)

16. J.E. Michaels, in Proceedings, Third U.S. National Congress on Applied Mechanics, ed. by R.M. Haythornthwaite, L.H. Donnell, M.M. Frocht (Brown University, Providence, 1958), p. 209

17. M. Dubois, F. Enguehard, L. Bertrand, Phys. Rev. E 50, 1548 (1994)

18. P.A. Doyle, J. Phys. D: Appl. Phys. 19, 1613 (1986)

19. N.N. Hsu, Dynamic Green's functions of an infinite plate - a computer program (Technical Report No. NBSIR 85-3234, National Bureau of Standards, Washington, 1985)

20. A.N. Ceranoglu, Y.-H. Pao, J. Appl. Mech. - T. Asme 48, 133 (1981)

21. J. Laloš, A. Babnik, J. Možina, T. Požar, Ultrasonics 66, 34 (2016)

22. T. Požar, P. Gregorčič, J. Možina, Appl. Phys. A 112, 165 (2013)

23. C.B. Scruby, R.J. Dewhurst, D.A. Hutchins, S.B. Palmer, J. Appl. Phys. 51, 6210 (1980)

24. T. Požar, J. Možina, in Fundamentals of Picoscience, ed. by K.D. Sattler (Taylor and Francis, Boca Raton, 2014), p. 553-577

25. J.-D. Aussel, J.-P. Monchalin, J. Appl. Phys. 65, 2918 (1989)

26. A.G. Every, Z.N. Utegulov, I.A. Veres, J. Appl. Phys. 114, 203508 (2013)

27. F. Enguehard, L. Bertrand, J. Acoust. Soc. Am. 103, 771 (1998)

28. T. Požar, P. Gregorčič, J. Možina, Opt. Express 17, 22906 (2009)

29. T. Požar, R. Petkovšek, J. Možina, Appl. Phys. Lett. 92, 234101 (2008) 